\title{
Conocimientos y prácticas de protección de los efectos nocivos de la radiación solar en estudiantes de colegios nacionales y par- ticulares del distrito de El Tambo 2015
}

\author{
Knowledge and practices to protect the harmful effects of solar radiation in stu- \\ dents of national and private colleges of the district of El Tambo 2015
}

Ana L.!. Girón y Richar, Barrera E!"

(1)Facultad de Enfermería, Universidad Nacional del Centro del Perú Email: analucila2@hotmail.com / richarin2701@hotmail.com

\section{Resumen}

La presente investigación es de tipo básico, descriptivo, correlacional; tuvo como problema general: ¿Cuál es la relación que existe entre los conocimientos y prácticas de protección de los efectos nocivos de la radiación solar en estudiantes de colegios nacionales y particulares del distrito de El Tambo 20 I5?, El objetivo de la investigación fue establecer la relación que existe entre los conocimientos y prácticas de protección de los efectos nocivos de la radiación solar en estudiantes de colegios nacionales y particulares del distrito de El Tambo 2015. La muestra estuvo conformada por 100 estudiantes de un colegio nacional y un colegio particular que se encuentran ubicados en el distrito del El Tambo. Al realizar la prueba estadística de correlación de Pearson, se encontró que existe una relación baja directa entre los conocimientos y prácticas de protección de los efectos nocivos de la radiación solar en estudiantes de colegios nacionales y particulares del distrito de El Tambo 2015 $(r=0,193$; valor $p<0.027)$. El nivel de conocimientos y prácticas sobre protección de los efectos nocivos de la radiación solar en estudiantes de colegios nacionales y particulares del distrito de El Tambo 20 I5, fue medio: de la misma manera no existe diferencia del nivel de conocimientos y practicas sobre protección de los efectos nocivos de la radiación solar entre varones y mujeres de colegios nacionales y particulares del distrito de El Tambo 2015. Concluyendo que los estudiantes de los dos colegios del distrito de EL Tambo, presentan conocimientos de nivel medio y práctica de nivel medio sobre la protección de los efectos nocivos de la radiación solar, los mismos que se encuentran relacionados de manera directa pero baja.

Palabras claves: conocimiento, prácticas, de autocuidado, efectos nocivos de la radiación solar

\begin{abstract}
This research is basic, descriptive, correlational; I had a general problem: What is the relationship between knowledge and practice of protection from the harmful effects of solar radiation on students of national schools and individuals in the district of El Tambo 2015?. The objective of the research was to establish the relationship between knowledge and practice of protection from the harmful effects of solar radiation in national schools students and individuals in the district of El Tambo 2015. The sample consisted of 100 students from a national school and a private school that are located in the district of El Tambo. When performing statistical test Pearson correlation, it was found that there is a direct low relationship between knowledge and practices of protection from the harmful effects of solar radiation on students of national schools and individuals in the district of El Tambo 2015 ( $r=0.193$ ; $p$ value $<0.027$ ). The level of knowledge and practices on protection from the harmful effects of solar radiation in national schools students and individuals in the district of El Tambo 20 I5, is medium. In the same way there is no difference in the level of knowledge and practical protection from the harmful effects of solar radiation between men and women in national schools and individuals in the district of El Tambo 2015. Concluding that students from the two scools in the district of El Tambo mid-level present knowledge and midlevel practices on protection of the harmful affects of solar radiation, the same that are directly related but low.
\end{abstract}

Keywords: knowledge, practices, self-care, harmful effects of solar radiation 


\section{Introducción}

El medio ambiente ha ido cambiando con el pasar del tiempo a causa de fenómenos naturales y por obra del consumo humano. Esta última se ha intensificado en el último siglo tanto así que afectó la capa de ozono llegando a producir su adelgazamiento e incluso un agujero.

El grosor de la capa de ozono interviene en el pasaje de radiación hacia la tierra, al estar adelgazándose permite que cada año se incremente el pasaje de radiación ultravioleta (RUV), dañina para la vida. Esta radiación produce daño a corto plazo que se aprecia como "quemadura solar" y a largo plazo como manchas, pecas, envejecimiento y cáncer de piel entre otros en el ser humano. La protección solar se encarga de prevenir los daños que causa la exposición a la radiación ultravioleta. Existen formas de protección física (capa de ozono, objetos barrera), química (fotoprotectores), biológicas (melanina) que se deben utilizar para evitar el daño por la RUV y la posterior aparición de cáncer de piel.

Los efectos sobre la piel inducidos por la exposición solar hacen en la actualidad interesante el abordaje del tema de protección solar, los conocimientos que de ésta tiene la población y la aplicación de estos conocimientos en la protección solar efectiva, de manera que se eviten a corto plazo eventos como la quemadura solar, a mediano plazo la pigmentación de las áreas expuestas y a largo plazo efectos como el foto envejecimiento, lesiones pre malignas como queratosis actínicas y malignas como carcinomas baso celulares y escamo celulares. (I)

El cáncer de piel es una enfermedad ubicada en tercer lugar de frecuencia según la Oranización Mundial de la Salud (OMS) y con alta morbilidad y mortalidad por lo que es necesario el conocimiento de sus causas y aplicación de medidas preventivas que detengan el incremento de su frecuencia en la población. El presente trabajo busca determinar el nivel de conocimientos y las prácticas que posee una población en especial, alumnos de los centros educativos del distrito de El Tambo.

\section{Materiales y métodos}

Por la naturaleza del problema se realizó una investigación de diseño no experimental, de tipo básico correlacional, debido a la naturaleza de nuestras variables y a la recolección de datos en un solo tiempo en dos colegios uno nacional y otro particular del distrito de El Tambo.

Se recopilaran los datos necesarios mediante encuesta sobre conocimientos y prácticas de protección de los efectos nocivos de la radiación solar.

Para la contrastación de la hipótesis se realizó con la prueba estadística $r$ de Pearson; para comprobar la hipótesis y medir los niveles de conocimientos y prácticas, se utilizó la prueba t de studente, para el procesamiento de la información se utilizó y el paquete estadístico SPSS versión 22.

\section{Resultados}

Para el presente estudio se obtuvieron los datos de 100 estudiantes

Tabla I.

Nivel de conocimientos de protección de los efectos nocivos de la radiación solar de los estudiantes de colegios nacionales y particulares del distrito de El Tambo 2015

\begin{tabular}{ccccccc} 
& $\mathrm{t}$ & $\mathrm{gl}$ & $\begin{array}{c}\text { Sig. } \\
\text { (bilateral) }\end{array}$ & $\begin{array}{c}\text { Diferencia } \\
\text { de medidas }\end{array}$ & $\begin{array}{c}95 \% \text { de intervalo de } \\
\text { confianza de la } \\
\text { diferencia } \\
\text { Inferior }\end{array}$ & Superior \\
\hline TOTAL & 6,7 & 99 &, 000 & 3,080 & 2,18 & 3,98 \\
\hline
\end{tabular}

\section{Tabla 2 .}

Nivel de prácticas de protección de los efectos nocivos radiación solar de los estudiantes de colegios nacionales particulares del distrito de El Tambo 2015

$\begin{array}{cccc}\begin{array}{c}\text { NIVEL DE } \\ \text { CONOCIMIENTOS }\end{array} & \text { FRECUENCIA } & \text { PORCENTAIE } & \begin{array}{c}\text { PORCENTAJE } \\ \text { VALIDO }\end{array} \\ \text { Bajo } & 20 & 20 & 20,0 \\ \text { Medio } & 61 & 61 & 81,0 \\ \text { Alto } & 19 & 19 & 100,0 \\ \text { Total } & 100 & 100 & \end{array}$

Fuente: Cuestionario de Conocimientos y prácticas de efectos nocivos de radiación solar.

Tabla 3.

Prueba " $t$ " de student para nivel de conocimientos sobre protección de los efectos nocivos de la radiación solar

\begin{tabular}{|c|c|c|c|c|c|c|}
\hline & \multicolumn{6}{|c|}{ VALOR DE LA PRUEBA $=31$} \\
\hline & \multirow[t]{2}{*}{ t } & \multirow[t]{2}{*}{$g \mid$} & \multirow[t]{2}{*}{$\begin{array}{c}\text { Sig. } \\
\text { (bilateral) }\end{array}$} & \multirow[t]{2}{*}{$\begin{array}{c}\text { Diferencia } \\
\text { de medidas }\end{array}$} & \multicolumn{2}{|c|}{$\begin{array}{l}95 \% \text { de intervalo de } \\
\text { confianza de la } \\
\text { diferencia }\end{array}$} \\
\hline & & & & & Inferior & Superior \\
\hline TOTAL & 6,7 & 99 & .000 & 3,080 & 2,18 & 3,98 \\
\hline
\end{tabular}

Tabla 4.

Prueba "t" de student para nivel de prácticas sobre protección de los efectos nocivos de la radiación solar

\begin{tabular}{|c|c|c|c|c|c|c|}
\hline & \multicolumn{6}{|c|}{ VALOR DE LA PRUEBA $=26$} \\
\hline & \multirow[t]{2}{*}{$\mathrm{t}$} & \multirow[t]{2}{*}{$\mathrm{g}$} & \multirow[t]{2}{*}{$\begin{array}{c}\text { Sig. } \\
\text { (bilateral) }\end{array}$} & \multirow[t]{2}{*}{$\begin{array}{c}\text { Diferencia } \\
\text { de medidas }\end{array}$} & \multicolumn{2}{|c|}{$\begin{array}{c}95 \% \text { de intervalo de } \\
\text { confianza de la } \\
\text { diferencia }\end{array}$} \\
\hline & & & & & Inferior & Superior \\
\hline TOTAL & 8,7 & 99 &, 000 & 5,520 & 4,27 & 6,77 \\
\hline
\end{tabular}




\section{Discusión}

El presente estudio se realizó en una población joven con edad promedió 15,30 \pm de I.010 años, siendo la población con mayor exposición. La población estuvo conformada en su mayoría por mujeres (66\%).

En la distribución según el colegio de procedencia se aprecia que los alumnos proceden en su mayoría de colegio estatal (57\%), estadísticamente no existe diferencia con el total de alumnos procedentes de colegio particular (43\%).

Con respecto al nivel de conocimiento sobre los efectos nocivos de la radiación solar los hallazgos nos indican que el $45 \%$ de los estudiantes de los colegios nacionales y particulares, presentan un nivel de conocimiento medio, sobre radiación solar (ultravioleta, efectos) y los fotoprotectores tópicos (bloqueador solar) y externos (uso de gorro, ropa de manga larga, gafas, búsqueda de áreas sombreadas) y $21 \%$ tienen un conocimiento bajo. Estos resultados contrastan con los hallazgos de Ramos, encontramos iguales resultados el 53\% tienen conocimiento es aceptable, así también respecto a la práctica de fotoprotección (uso de gorro, lentes, bloqueador solar, vestimenta con mangas largas, búsqueda en horas de mayor radiación solar) muestran resultados $61 \%$ realizan un nivel de prácticas media, similares hallazgos se encontraron por Ramos, con que el 59.1\% tienen practicas adecuadas, así también podemos ver que Loza, indica que el $60 \%$ de los estudiantes tiene un nivel inadecuado de prácticas de fotoprotección.

\section{Conclusiones}

I.- Existe relación directa pero abaja entre los niveles de conocimientos y prácticas de protección de los efectos nocivos de la radiación solar en estudiantes de colegios nacionales y particulares del distrito de El Tambo 20 I 5.

2.- El nivel de conocimientos sobre conocimientos de protección de los efectos nocivos de la radiación solar es de nivel medio.

3.- El nivel de prácticas sobre prácticas de protección de los efectos nocivos de la radiación solar es de nivel medio.

4.- No existe diferencia de conocimientos y prácticas sobre os efectos nocivos de la radiación solar en estudiantes varones y mujeres.

\section{Referencias}

Página web, http://www.ronzon.com/RUV.htm

Batista, T. (20/3). Assessment of sun protection and skin cáncer prevention among preschool children. Rev Paul Pediatr; 3 I ( I): I7-23.

Stankeviciutex, V., Zaborskis, A; Petrauskienex, A. \& Valiukeviciene, S. Skin. (2004). Cancer prevention: children's health education on protection from sun exposure and assessment of its efficiency. Medicina (Kaunas) ; 40(4):386-393.

Laffargue, J. A. (20II). Encuesta sobre protección solar en adolescentes deportistas de la Provincia de Buenos Aires. Arch Argent Pediatr; 109( I):30-35. 